\title{
Evaluating the Effect of Repetitive Transcranial Magnetic Stimulation (rTMS) and Humanistic Care Treatment on Cognitive Impairment Patients
}

\author{
Yingxue Zhong ${ }^{1}$, Danfeng Jiang ${ }^{2,}$, Guoqiang Zheng ${ }^{1}$ \\ ${ }^{1}$ Department of Renal Rehabilitation, the First Affiliated Hospital, Jinan University, Guangzhou, China \\ ${ }^{2}$ Psychology Department, the First Affiliated Hospital, Jinan University, Guangzhou, China \\ Email address: \\ Yingxuezhong456@163.com (Yingxue Zhong), Danfengjiang456@163.com (Danfeng Jiang), \\ guoqiangzheng456@163.com (Guoqiang Zheng) \\ *Corresponding author

\section{To cite this article:} \\ Yingxue Zhong, Danfeng Jiang, Guoqiang Zheng. Evaluating the Effect of Repetitive Transcranial Magnetic Stimulation (rTMS) and \\ Humanistic Care Treatment on Cognitive Impairment Patients. American Journal of Psychiatry and Neuroscience. \\ Vol. 8, No. 2, 2020, pp. 30-32. doi: 10.11648/j.ajpn.20200802.12
}

Received: April 29, 2020; Accepted: May 18, 2020; Published: June 4, 2020

\begin{abstract}
Objective: To evaluate the effect of repetitive transcranial magnetic stimulation (rTMS) and Humanistic care treatment on Cognitive impairment patients. Methods: We invested 60 patients to join our study who were diagnosed as cerebrovascular disease or alzheimer disease, the diseases are cognitive impairment. Their diagnose time is from January 2019 to December 2019. We randomly assign the participants to two groups, that include control group $(n=30)$ and intervention group $(\mathrm{n}=30)$. In control group, participants receive conventional responsibility mode of nursing. Intervention group participants receive humanistic care model in treatment process. Result: In Unplanned extubation rate of rTMS, the control group has more case of Unplanned extubation, it also has higher Unplanned extubation rate than that of intervention group [3 (10.0\%) vs 7 (23.3\%)]. In incidence of agitation, it shows the cases of intervention group are less than that of control group, and it is statistical significance ( $8 \mathrm{vs} 13, \mathrm{p}=0.014)$. In accurate response research, the intervention group has better performance in accurate response test, its accurate response rate is $86.7 \%$ and the case of accurate response is 26 , that is better than that of control group in our research result. Conclusion: The effect of repetitive transcranial magnetic stimulation (rTMS) and Humanistic care treatment is good for improve treatment outcome of cognitive impairment.
\end{abstract}

Keywords: Cognitive Impairment, rTMS, Humanistic Care

\section{Introduction}

Repetitive transcranial magnetic stimulation (rTMS) is a non-invasive brain stimulation technique. It is often used to treat several psychiatric and neuro-psychiatric disorders, such as depression, PTSD, migraine, etc [1,2]. In addition, most of the studies till date have evaluated the effects of $10 \mathrm{~Hz}$ pulses, and it can deliver up to 20,000 pulses to the brain in shorter treatment protocols [3-5]. In some report, however, higher frequency treatments over longer times and higher number of total magnetic pulses administered have better improvements in the result [6]. But its advantages cannot be ignored, the rTMS is non-invasively and is free from drug-related side effects. Hallett's report indicated that rTMS can be effective in the management of refractory neuropathic pain [7, 8]. For instance, primary motor cortex stimulation with rTMS can effectively influence drug-resistant pain and refractory neuropathic pain arising from peripheral nerve [9]. Base on some reports, rTMS may exert therapeutic value have been hypothesized, such as inducing changes in cerebral blood flow, neuro-endocrine effects, neurotransmitter levels, and connectivity between different brain regions [10-12].

cognitive impairment is a common morbidity, it not only had high prevalence, but also had maximum impact on the quality of life. Even Mild cognitive impairment predisposes the person to development of dementia in future [13]. Prevalence of cognitive impairment increases with age and is projected to increase with rising life expectancy in developed as well as 
developing nations. According to an India's study, people who older than 80 years of age had high prevalence of dementia (15\%), but people who were less than 60 years only had $2.6 \%$ of prevalence of dementia [14]. Cognitive impairment also is a growing public health concern throughout the world. It can cause a series of problems, that increase the risk of falls and dis-ability and worsen the health-related quality of life in older adults [15]. The objective of this study is that assess the effect of repetitive transcranial magnetic stimulation and humanistic care treatment on cognitive impairment patients.

\section{Methods}

\subsection{Participants Enrollment and Survey Methods}

We invested 60 patients to join our study who were diagnosed as cerebrovascular disease or alzheimer disease, the diseases are cognitive impairment. Their diagnose time is from January 2019 to December 2019. We randomly assign the participants to two groups, that include control group $(n=30)$ and intervention group $(n=30)$. The two group participants receive different nursing model. In control group, participants receive conventional responsibility mode of nursing. Intervention group participants receive humanistic care model in treatment process. The humanistic care included that new communication skills, psychological assessment of patients and new nursing measure. Our research use hospital record, simple questionnaire and interview to collect the data, the data include Pipe removal rate, Incidence of agitation and Accurate response rate.

About humanistic care model, treatment visits were conducted at selected time, and self-introduction was initiated to the patients during the visits, that it provides good image to patients and improve the sense of trust of patients. In addition, we provide the information was associated with rTMS and cognitive impairment to patients or their guardians. Base on patient situation, we encourage patients to ask relevant questions, and their emotion are relaxed as we answer their questions. In communication process, we use suitable communication skills to talk with patients and their guardians.

Their inclusion criteria were: (1) The patients were diagnosed as cerebrovascular disease or alzheimer disease; (2) Patients volunteered to participate our study in treatment. Their withdraw criteria were: (1) The patient's family disagreed that; (2) Sick again and again.

\subsection{Statistical Analysis}

Our data analyzer performed the statistical analysis by SPSS 22.0. The $\mathrm{P}$ value, t-test and chi-square test were associated with collection result were analyzed. Besides, the mean standard deviation for statistical description.

\section{Result}

The Table 1 shows the unplanned extubation rate of rTMS in our research result. The control group has more case of Unplanned extubation, it also has higher Unplanned extubation rate than that of intervention group [3 $(10.0 \%)$ vs 7 $(23.3 \%)]$. Additionally, the result is statistical significance.

Table 1. Unplanned extubation rate of rTMS.

\begin{tabular}{lll}
\hline Projects & $\begin{array}{l}\text { Case of Pipe } \\
\text { removal }(\mathbf{n})\end{array}$ & $\begin{array}{l}\text { Pipe removal rate } \\
(\%)\end{array}$ \\
\hline Intervention group $(\mathrm{n}=30)$ & 3 & $10.0 \%$ \\
Control group $(\mathrm{n}=30)$ & 7 & $23.3 \%$ \\
$\mathrm{~T}$ & 5.172 & 4.091 \\
P value & $<0.005$ & $<0.005$ \\
\hline
\end{tabular}

In table 2, the research result indicates participants' incidence of agitation in the rTMS. The Case of agitation of intervention group is less than that of control group, and it is statistical significance ( 8 vs $13, \mathrm{p}=0.014)$. In addition, intervention group has lower incidence of agitation $(26.7 \% \mathrm{vs}$ $43.3 \%)$.

Table 2. Incidence of agitation.

\begin{tabular}{lll}
\hline Projects & $\begin{array}{l}\text { Case of agitation } \\
\text { (n) }\end{array}$ & $\begin{array}{l}\text { Incidence of } \\
\text { agitation (\%) }\end{array}$ \\
\hline Intervention group $(\mathrm{n}=30)$ & 8 & $26.7 \%$ \\
Control group $(\mathrm{n}=30)$ & 13 & $43.3 \%$ \\
$\mathrm{~T}$ & 3.562 & 5.471 \\
P value & 0.014 & $<0.005$ \\
\hline
\end{tabular}

Accurate response also is judgment standard of Cognitive impairment situation in treatment process (Table 3). The intervention group has better performance in accurate response test, its accurate response rate is $86.7 \%$ and the case of accurate response is 26 , that is better than that of control group in our research result.

Table 3. Accurate response rate.

\begin{tabular}{lll}
\hline Projects & $\begin{array}{l}\text { Case of Accurate } \\
\text { response (n) }\end{array}$ & $\begin{array}{l}\text { Accurate response } \\
\text { rate }(\%)\end{array}$ \\
\hline Intervention group $(\mathrm{n}=30)$ & 26 & $86.7 \%$ \\
Control group $(\mathrm{n}=30)$ & 20 & $66.7 \%$ \\
$\mathrm{~T}$ & 6.781 & 11.933 \\
P value & $<0.005$ & $<0.005$ \\
\hline
\end{tabular}

\section{Discussion and Conclusion}

rTMS have been demonstrated in obstructive sleep apnea syn-drome, restless legs syndrome (RLS), insomnia, and sleep deprived healthy subjects; moreover, rTMS techniques may provide a further understanding on the role of neurotransmission pathways and plastic remodeling of neuronal networks involved in common sleep disorders [16, 17]. In addition, the report indicated the inactive placebos produced improvement that was $75 \%$ of the effect of the active antidepressants and that of $64 \%$ in hypnotic [18]. In other define, rTMS is typically defined as a failure to respond to at least 2 trials of antidepressant medications. From some multiple meta-analyses of randomised clinical trial, the effectiveness of rTMS is supported [19].

According to above result, the effect of repetitive transcranial magnetic stimulation (rTMS) and Humanistic care treatment is good for improve treatment outcome of cognitive impairment. In ripe removal rate of rTMS, the intervention group 
participants have better performance in our study, they have lower pipe removal rate than control group. In incidence research of agitation, intervention group has lower incidence of agitation, it indicates that rTMS and humanistic care treatment have good improvement to cognitive impairment patients. The accurate response research, it shows intervention group has better performance in accurate response test, its accurate response rate is $86.7 \%$ and the case of accurate response is 26 . The rTMS and humanistic care treatment also have good improvement to increase accurate response rate.

\section{References}

[1] Stilling JM, Monchi O, Amoozegar F, Debert CT. Transcranial magnetic and direct current stimulation (TMS/tDCS) for the treatment of headache: a systematic review. Headache J Head Face Pain. 2019; 59 (3): 339-357.

[2] Yan T, Xie Q, Zheng Z, Zou K, Wang L. Different frequency repetitive transcranial magnetic stimulation (rTMS) for posttraumatic stress disorder (PTSD): a systematic review and meta-analysis. J Psychiatr Res. 2017; 89: 125-135.

[3] Kennedy NI, Lee WH, Frangou S. Efficacy of non-invasive brain stimulation on the symptom dimensions of schizophrenia: a meta-analysis of randomized controlled trials. Eur Psychiatr. 2018; 49: 69-77.

[4] Aleman A, Enriquez-Geppert S, Knegtering H, Dlabac-de Lange JJ. Moderate effects of noninvasive brain stimulation of the frontal cortex for improving negative symptoms in schizophrenia: meta-analysis of controlled trials. Neurosci Biobehav Rev. 2018; 89: 111-118.

[5] He H, Lu J, Yang L, et al. Repetitive transcranial magnetic stimulation for treating the symptoms of schizophrenia: a PRISMA compliant meta-analysis. Clin Neurophysiol. 2017; 128 (5): 716-724.

[6] Rabany L, Deutsch L, Levkovitz Y. Double-blind, randomized sham controlled study of deep TMS add-on treatment for negative symptoms and cognitive deficits in schizophrenia. J Psychopharmacol. 2016; 28 (7): 686-690.

[7] Khedr EM, Kotb H, Kamel NF, et al. Long-lasting antalgic effects of daily sessions of repetitive transcranial magnetic stimulation in central and peripheral neuropathic pain. $J$ of Neurol, Neurosurg, and Psychiatry. 2015; 76: 833-838.

[8] Hallett M. Transcranial magnetic stimulation: Neuron. 2017; 55: $187-99$.
[9] Connell NE, Marston L, Spencer S, et al. Non-invasive brain stimulation techniques for chronic pain. Cochrane Database Syst Rev. 2018; 4: CD008208.

[10] Lan MJ, Chhetry BT, Liston C, Mann JJ, Dubin M. Transcranial magnetic stimulation of left dorsolateral prefrontal cortex induces brain morphological changes in regions associated with a treatment resistant major depressive episode: an exploratory analysis. Brain Stimul. 2016; 9 (4): 577-583.

[11] Noda Y, Silverstein WK, Barr MS, Vila-Rodriguez F, Downar J, Rajji TK, et al. Neurobiological mechanisms of repetitive transcranial magnetic stimulation of the dorsolateral prefrontal cortex in depression: a systematic review. Psychol. Med. 2015; 45 (16): 3411-3432.

[12] He Y, Li K, Chen Q, Yin J, Bai D. Repetitive transcranial magnetic stimulation on motor recovery for patients with stroke: a PRISMA compliant systematic review and metaanalysis. Am. J. Phys. Med. Rehabil. 2019; 99 (2): 99-108.

[13] Sharma D, Mazta S, Parashar A. Prevalence of cognitive impairment and related factors among elderly: a population-based study. J. Dr. NTR Univ. Heal. Sci. 2016; 2: 171.

[14] Poddar K, Kant S, Singh A, Singh TB. An epidemiological study of dementia among the habitants of eastern Uttar Pradesh, India. Ann. Indian Acad. Neurol. 2015; 14: 164-168.

[15] Shimada H, Makizako H, Doi T, Tsutsumimoto K, Lee S, \& Suzuki T. Cognitive impairment and disability in older japanese adults. PloS One. 2016; 11 (7): e0158720.

[16] Jiang CG, Zhang T, Yue FG, et al. Efficacy of repetitive transcranial magnetic stimulation in the treatment of patients with chronic primary insomnia. Cell Biochem Biophys 2013; 67 (1): 169-173.

[17] Lanza G, Cantone M, Lanuzza B, et al. Distinctive patterns of cortical excitability to transcranial magnetic stimulation in obstructive sleep apnea syn-drome, restless legs syndrome, insomnia, and sleep deprivation. Sleep Med Rev 2015; 19: 39-50.

[18] Furukawa TA, Cipriani A, Atkinson LZ, et al. Placebo response rates in anti-depressant trials: a systematic review of published and unpublished double-blind randomised controlled studies. Lancet Psychiatry 2016; 3 (11): 1059-1066.

[19] Brunoni AR, Chaimani A, Moffa AH, Razza LB, Gattaz WF, Daskalakis ZJ, et al. Repetitive transcranial magnetic stimulation for the acute treatment of major depressive episodes: a systematic review with network meta-analysis. JAMA Psychiatry. 2017; 74: 143-152. 